\title{
Index autorum ad Vol. 157
}

\section{Confecit: C. Loeb-Schürch}

(B) $=$ Buchbesprechungen - Book revie $\lambda v s-$ Livres nouveaux $(V)=$ Vortrag - Report Communication

Alessio, L., 343 Amemiya, T., 462

Becker, 83 (B)

Békésy, G. von, v. Von Békésy, G.

Bertoni, G., 343

Betlelheim, H., 1

Blagojevic, M., 14

Blumenthal, M., 274

Caldeira, J. A. F., 279 Casanovas, J., 163 (B) Castellazzo, R., 43

Charamis, J., 407 Cibis, P. A., 84 (B) Contino, F., 86 (B) Coscas, G, 399 (B) Csapody, I., 131

Dake, C. L., 88 (B)

De Decker, W., 142

Dieckhues, B., 398 (B)

Dolenek, 161 (B)

Drews, 83 (B)

Dufour, 162 (B), 165 (B)

Dymitrowska, Maria, 119, 306

Index autorum ad Vol. 157

483

Etzine, S., 263

Fasanella, R. M., 399 (B)

Ferrer, Olga, 89

Fiedler, H., 178

Fison, 162 (B), 165 (B)

Fötzsch, R., 328

Francois, J., 83 (B), 87 (B), 88 (B),

231 Franke, G., 374 Friedrich, J., 301 Freund, H. R., 165 (B)

Gálfi, I., 349 Gall, J., 154 Gartner, J., 414 Gernet, 399 (B) Giesbrecht, Astrea M., 279 Giessman, H. G., 374 Gil de Rio, E., 400 (B) Girard, J., 166 (B) Gloeckner, S, 165 (B) Goder, G., 400 (B) Goldschmidt, M., 381 Gombos, G. M., 274 (B) Gramberg-Danielsen, B., 162 (B) Grignolo, A., 43

Havener, W. H., 165 (B) Heszberger, S., 391 Hochwin, O, 87 (B) Holzki, Ursula, 142 Horsten, G. P. M., 293

Jessen, K., 60 Jobbágyi, P., 391 Jordano, J., 223 Jütte, A., 169

Kaufmann, Trudy, 249 Katzin, H. M., 400 (B) Kazanowska, W., 119, 306 Kazner, E., 164 (B)

Kern, R., 65 Kloucek, F., 442 
Lańcucki, J., 357 Lauber, Ursula, 142 Leira, H., 161 (B) Lemke, L., 169 Lerche, W., 451 Liegl, O., 362

Liesenfeld, H. J., 161 (B) Lombardi, G., 321

Maciejewska, J., 119, 306

Magora, A., 110

Malbran, J., 268

Mannucci, P. M., 343

McLean, J. M., 165 (B)

Mehlhose, F, 201

Merz, M., 357

Meyer-Schwickerath, 162 (B), 165 (B)

Mrzyglód, S., 313

Nover, A., 414

Oberhoff, P., 87 (B) Oksala, 399 Opitz, J., 169 Orzalesi, N., 43 Otradovec, J., 427, 442 Otto, J., 135

Paliaga, G. P., 166 (B) Pau, H., 206 Paunescu, Eugenia, 193 Piscevic, 14 Poeck, K., 83 (B)

Postic, S., 398 (B)

Quesada, F., 223

Radian, A. B., 193 Radian, Ana Lucia, 193 Radosavljevic, M., 14 Ramos, A. O., 279 Ramos, L., 279 Rempt, F., 104 Réthy, Mácia, 285 Richter, Susanne, 163 (B) Roper-Hall, 164 (B) Rousselie, F., 399 (B)

Sachsenweger, 83 (B) Sakaue, E., 462 Sampaolesi, R., 86 (B) Saraux, H., 399 (B) Sautter, 164

(B) Schiefer, W., 164 (B) Schlote, H. W., 374 Sebestyén, J., 349 Sedan, J., 36 Segal, P., 313

484

Index autorum ad Vol. 157

Shikano, S., 167 (B) Shimizu, K., 167 (B) Slangier, E., 135 Stefanovic, B., 14 Streiff, 164 (B) Szmigielski, M., 357

Takac, 161 (B) Théodossiadis, G., 407 Todter, F., 76 Toril, M., 223 Toselli, C, 343 Trzcinska-

Dabrowska, Zofia, 24

Varga, Margit, 154 Verrey, F., 401

Vilanova, X., 163 (B)

Vit, H., 76

Vittone, P., 43

Von Békésy, G., 163 (B)

W. Wainstein, R., 231 Weigelin, E., 60, 87 (B) Wessing, A., 85 (B) Wilson, G., 400 (B)

Winkelman, J. E., 293 Wittwer, B., 178 Witwicki, T., 24 Worst, J. G. F., 88 (B) Wulle, K. G., 451

Zaubermann, H., 110 Zielinska, Krystyna, 24 